\title{
A Comprehensive Sequencing-Based Analysis of Allelic Methylation Patterns in Hemostatic Genes in Human Liver
}

\author{
Martina Olsson Lindvall ${ }^{10}$ Marcela Davila Lopez ${ }^{2}$ Sofia Klasson ${ }^{1}$ Lena Hansson ${ }^{3,4}$ Staffan Nilsson ${ }^{1,5}$ \\ Tara M. Stanne ${ }^{1, *}$ Christina Jern ${ }^{1,6, *}$
}

${ }^{1}$ Department of Laboratory Medicine, Institute of Biomedicine, Sahlgrenska Academy at University of Gothenburg, Gothenburg, Sweden

2 Bioinformatics Core Facility, University of Gothenburg, Gothenburg, Sweden

${ }^{3}$ Science for Life Laboratories (SciLifeLab), Stockholm, Sweden

${ }^{4}$ Novo Nordisk, Oxford, United Kingdom

5 Department of Mathematical Sciences, Chalmers University of Technology, Gothenburg, Sweden

${ }^{6}$ Department of Clinical Genetics and Genomics, Sahlgrenska

University Hospital, Gothenburg, Sweden

\author{
Address for correspondence Martina Olsson Lindvall, MSc, Institute \\ of Biomedicine, Sahlgrenska Academy at the University of \\ Gothenburg, Box 445, SE-405 30 Gothenburg, Sweden \\ (e-mail: martina.olsson@gu.se).
}

Thromb Haemost 2020;120:229-242.

\begin{abstract}
Keywords

- coagulation factors

- gene regulation

- hemostasis

- single-nucleotide polymorphisms

Characterizing the relationship between genetic, epigenetic (e.g., deoxyribonucleic acid [DNA] methylation), and transcript variation could provide insights into mechanisms regulating hemostasis and potentially identify new drug targets. Several hemostatic factors are synthesized in the liver, yet high-resolution DNA methylation data from human liver tissue is currently lacking for these genes. Single-nucleotide polymorphisms (SNPs) can influence DNA methylation in cis which can affect gene expression. This can be analyzed through allele-specific methylation (ASM) experiments. We performed targeted genomic DNA- and bisulfite-sequencing of 35 hemostatic genes in human liver samples for SNP and DNA methylation analysis, respectively, and integrated the data for ASM determination. ASM-associated SNPs (ASM-SNPs) were tested for association to gene expression in liver using in-house generated ribonucleic acid-sequencing data. We then assessed whether ASM-SNPs associated with gene expression, plasma proteins, or other traits relevant for hemostasis using publicly available data. We identified 112 candidate ASM-SNPs. Of these, $68 \%$ were associated with expression of their respective genes in human liver or in other human tissues and $54 \%$ were associated with the respective plasma protein levels, activity, or other relevant hemostatic genome-wide association study traits such as venous thromboembolism, coronary artery disease, stroke, and warfarin dose maintenance. Our study provides the first detailed map of the DNA methylation landscape and ASM analysis of hemostatic genes in human liver tissue, and suggests that methylation regulated by genetic variants in cis may provide a mechanistic link between noncoding SNPs and variation observed in circulating hemostatic proteins, prothrombotic diseases, and drug response.
\end{abstract}

\footnotetext{
* Authors contributed equally.
}

received

May 28, 2019

accepted after revision November 1, 2019
DOI https://doi.org/ $10.1055 / \mathrm{s}-0039-3401824$. ISSN 0340-6245. (c) 2020 Georg Thieme Verlag KG Stuttgart . New York
License terms

(1) (1) $\odot \circledast$ 


\section{Introduction}

Plasma concentrations of hemostatic proteins vary in healthy humans and several hemostatic factors are synthesized in the liver. Characterizing the complex relationship between genetic, epigenetic (e.g., deoxyribonucleic acid [DNA] methylation), and transcriptomic variation has the potential to increase understanding about the mechanisms regulating hemostasis and to inform drug development. DNA methylation profiles vary among tissues and cell types, thus analyzing the appropriate tissue is essential. Genetic variants can exert an influence on DNA methylation in cis $^{1,2}$ which can then affect cis-regulatory gene expression., ${ }^{3,4}$ Consequently, it has been suggested that DNA methylation could provide the link between noncoding genetic variants and phenotypic variation.

DNA methylation at cytosine-guanine dinucleotides (CpGs) is the most studied epigenetic trait and has been most extensively studied in CpG islands in the promoter of genes, where it is widely regarded as a mechanism of transcriptional repression. ${ }^{5,6}$ More recently, however, DNA methylation within gene bodies (i.e., exons and introns) has been reported to be prevalent and to increase gene expression. ${ }^{7-10}$ Thus, the relationship between DNA methylation and gene expression is highly complex and not fully understood. ${ }^{11,12}$ Most methylation studies have used microarrays that cover $<4 \%$ of human $\mathrm{CpG}$ sites, and this percentage is mainly biased toward CpGs in promoters. ${ }^{13,14}$ The current gold standard method to investigate DNA methylation patterns is bisulfite conversion of the DNA followed by DNA sequencing, which allows determination of methylation patterns at a single-cytosine resolution. ${ }^{13,14}$ Despite the potential of DNA methylation studies to contribute to new knowledge on gene regulation, highresolution DNA methylation data are currently lacking for hemostatic genes in the liver.

Methylation quantitative trait loci (mQTL) mapping studies, which link variations in genotypes to DNA methylation levels at specific CpG sites across unrelated individuals, is one approach to elucidate whether genetic variants correlate to methylation of genes. Since DNA methylation is also influenced by environmental factors (e.g., smoking, diet, and drugs), large sample sizes are required. As a result, most MQTL studies to date have been performed in easy to access tissues such as blood, which is not necessarily the tissue of relevance. Furthermore, as most mQTL studies have used microarrays, our understanding of the roles of DNA methylation within gene bodies is currently limited. ${ }^{15,16}$

An alternative approach to MQTL is to compare methylation levels at specific CPG sites between each of the two alleles in the same individual. This alternative approach, referred to as allele-specific methylation (ASM), standardizes environmental and other confounding variables. Therefore, this is a powerful methodology to map putative cis-acting single-nucleotide polymorphisms (SNPs) even when using a small number of samples, ${ }^{17}$ which facilitates analysis of "hard to access" human tissues of relevance such as liver. The ASM approach requires genotyping of SNPs for differentiating the alleles and simultaneous measurements of DNA methylation at individual $\mathrm{CpG}$ sites. Both of these can be achieved by using targeted next-generation sequencing, which allows for the unbiased detection of all CpGs, including those within the gene body.

To identify novel regulatory elements involved in the control of hemostatic gene expression in liver we performed DNA and methylation sequencing of 35 hemostatic genes. As far as we are aware, no study has yet reported DNA methylation patterns on an individual CpG level nor analyzed ASM in hemostatic genes in human liver tissue samples. Given this background, the aims of the present study are to: (1) profile methylation patterns across hemostatic genes in human liver tissue using targeted bisulfite-sequencing; (2) identify ASMassociated SNPs (ASM-SNPs) in hemostatic genes in human liver; and (3) determine whether DNA methylation regulated by genetic variation could be a mechanistic link between noncoding SNPs and variation observed in gene expression, circulating hemostatic proteins, or other relevant traits.

\section{Methods}

\section{Sample Collection and Targeted Sequencing}

Tissue collection, genomic DNA (gDNA) and messenger ribonucleic acid (mRNA) extraction, and the target sequencing (-seq) designs for gDNA and mRNA have been previously described. ${ }^{18}$ In short, macroscopically noncirrhotic and nontumorous human liver tissue and blood samples were collected from adult patients of European descent $(n=19)$ undergoing liver surgery at the Sahlgrenska University Hospital, Gothenburg, Sweden. The institutional review board, i.e., the Ethics Committee of the University of Gothenburg approved the collection of the human liver tissues and blood samples, and their subsequent use for the purpose of this study. Clinical characteristics of study participants are summarized in - Supplementary Table $\mathbf{S 1}$ (available in the online version). gDNA was isolated from blood for genotyping (DNA-seq). gDNA and mRNA were simultaneously isolated from the same piece of liver tissue for methylation (bisulfiteseq) and expression (mRNA-seq) analysis, respectively.

We selected 35 genes that are predominantly expressed in the liver and are important for hemostasis by studying the literature, the Kyoto Encyclopedia of Genes and Genomes pathway database, and the Molecular Signatures Database (MSigDB, Broad Institute). Custom capture kits (Agilent, Santa Clara, California, United States) were designed for targeted sequencing. For full details on gDNA and mRNA sequencing, data processing, and analysis, please see Olsson Lindvall et al. ${ }^{18}$

\section{Targeted Gene Panel for Bisulfite-Seq}

A SureSelect custom capture kit (Agilent) was designed for DNA methylation analysis (bisulfite-seq) targeting the same 35 genes as for gDNA using Agilent's SureDesign tool (Agilent). Probes were designed to capture all exons, introns, and all potential upstream ( $\geq 5 \mathrm{~kb})$ and downstream $(0.5 \mathrm{~kb})$ regulatory regions, resulting in a 3.3-Mb design. Detailed information of all regions included in this study is shown in -Supplementary Table $\mathbf{S 2}$ (available in the online version). gDNA samples from liver were sheared to an average size of 
250 base pair (bp) and bisulfite converted with the EZ DNA methylation kit (Zymo Research, Irvine, California, United States). The bisulfite-treated DNA was then used to prepare strand-specific sequencing libraries using the customdesigned SureSelect kits (Agilent) according to the manufacturer's protocols. All sample libraries were multiplexed in one sequence lane on the Illumina HiSeq 2500 with high output mode and $100 \mathrm{bp}$ paired-end reads (Illumina, San Diego, California, United States), following the manufacturer's protocols. Approximately $15 \mathrm{M}$ reads were generated per sample (for coverage per gene see - Supplementary Fig. S1, available in the online version). Samples from two patients were also sequenced using SureSelect Methyl-Seq target enrichment system for Illumina (a commercially available kit from Agilent which provides targeted coverage of $84 \mathrm{Mb}$ of the human genome) to identify possible weaknesses or biases in our custom-designed kit (prepared as above). Assessment of the data showed no bias in DNA methylation values between the two kits, but as expected, coverage was much higher in our custom targeted-seq samples ( - Supplementary Fig. S1, available in the online version). All raw output sequence reads were quality controlled using FastQC (https://www.bioinformatics.babraham.ac.uk/projects/fastqc/). After removal of low-quality bases (Phred score $<30$ ), reads were trimmed (removal of adaptors and indexes) with Trim Galore! (http:// www.bioinformatics.babraham.ac.uk/projects/trim_galore/) and aligned to the human reference genome (GRCh37/hg19) with Bowtie2. ${ }^{19}$ Deduplication and methylation extraction were performed with the Bismark pipeline (https://www. bioinformatics.babraham.ac.uk/projects/bismark/) using default parameters. Finally, SeqMonk (https://www.bioinformatics.babraham.ac.uk/projects/seqmonk/) was used for visualization.

\section{Detection of ASM}

ASM analysis relies on the presence of heterozygous SNPs on the same read as a given $\mathrm{CpG}$ site to separate alleles prior to analysis. DNA methylation levels at individual $\mathrm{CpG}$ sites between the two alleles are then directly compared. Bisulfite-seq reads were aligned to the GRCh37/hg19 reference genome using Bowtie2. Known SNP positions in the reference genome were masked by the ambiguity base "N" prior to alignment to avoid read mapping biases. SNPsplit (https:// www.bioinformatics.babraham.ac.uk/projects/SNPsplit/) was then used to determine the allelic origin of reads overlapping SNP positions using individual-specific genotype information from the gDNA-seq data. Thus, the data was split into two in silico allele-specific aligned genomes. The methylation status of $\mathrm{CpGs}$ was then individually called from the two separate alignments using Bismark and the methylation level of each $\mathrm{CpG}$ was compared between the two alignments. To appropriately estimate methylation differences, we required a minimum of 30 reads per allele. CpGs were defined as ASM sites if at least one individual had a percent methylation difference of $>20 \%$ between the two alleles. The individual ASM differences were weighted by their inverse variances and then summed. The sum was finally scaled by its standard error to form a $Z$-statistic. A p-value of less than 0.05 was considered statistically significant. A positive percentage difference value indicates that the major allele is more methylated than the minor allele.

\section{Evaluation of ASM Enrichment in Different Genomic Contexts}

We used the right-handed Fisher's exact test to evaluate if CpGs displaying ASM were enriched in specific genomic contexts compared with the total number of $\mathrm{CpGs}$ in the same regions. The regions investigated for ASM enrichment were upstream regions (defined as $+5 \mathrm{~kb}$ to $+2 \mathrm{~kb}$ from transcription start site [TSS]), promoters (defined as $+2 \mathrm{~kb}$ to $-1 \mathrm{~kb}$ from TSS), first exon, remaining exons (excluding the first exon), introns, exon/intron boundaries, $\mathrm{CpG}$ islands, and $\mathrm{CpG}$ island shores. A p-value of less than 0.01 was considered statistically significant.

\section{Tag-SNP Extraction from Methyl-Seq Data}

Heterozygous SNPs on the same sequencing read as the CpG sites displaying ASM were extracted from the gDNA data. For CpGs with several heterozygous SNPs on the same read, the closest ones (up to three SNPs per $\mathrm{CpG}$ ) were selected.

\section{Association of ASM-SNPs to mRNA Expression Levels in RNA-Seq Data}

Given that DNA methylation is linked to the regulation of gene expression, we assessed whether a difference in allelic mRNA expression could be identified based on the ASMSNPs. Results from targeted RNA-seq in the same 19 individuals have previously been reported. ${ }^{18}$ Normalized estimates of gene expression, that is, transcripts per million were calculated per gene. We then evaluated all ASM-SNPs for association to expression levels by linear regression analysis using additive models in SPSS for Windows version 20 (IBM Corporation, New York, United States). The statistical significance cutoff (two-tailed) was 0.05 . As this study is based on only 19 samples, we are underpowered for interindividual analyses and therefore also report trends (defined as $p<0.2$ ).

\section{Association of ASM-SNPs to mRNA Expression in Publicly Available Expression Quantitative Trait Loci Data}

We next queried all ASM-SNPs and high linkage disequilibrium (LD) proxies ( $r^{2} \geq 0.8$ in 1,000 genomes European panel) against publicly available expression quantitative trait loci (eQTL) data using PhenoScanner (http://www.phenoscanner.medschl.cam. ac.uk; assessed May 2019) ${ }^{20}$ which includes results from the Genotype-Tissue Expression project v6 (http://www.gtexportal.org/home/datasets), ${ }^{21,22}$ NHLBI GRASP v2,, ${ }^{23}$ STARNET, ${ }^{24}$ and other smaller studies (see http://www.phenoscanner.medschl. cam.ac.uk/studies/ for details). eQTL results were filtered to retain only variants with $p<1 \times 10^{-5}$ and manually curated to include only eQTLs correlating to the 35 genes of interest.

\section{ASM-SNPs: Association with Circulating Proteins and Disease Traits}

To identify ASM-SNPs associated with circulating plasma levels or activity of the respective proteins and relevant 
disease and nondisease phenotypes, we queried our variants and their corresponding proxies $\left(r^{2} \geq 0.8\right)$ against publicly available genome-wide association study (GWAS) data using both the PhenoScanner "Proteins" and "Diseases \& traits" search functions (assessed May 2019) which includes results from the NHGRI-EBI GWAS catalog (https://www.ebi.ac.uk/gwas/) and the UK Biobank (http://www.ukbiobank.ac. $\mathrm{uk})$. For GWAS, summary statistics were filtered to $p<5 \times$ $10^{-5}$ and then manually curated to retain only entries with relevant traits. For ASM-SNPs in CPB2 and HAPB2, we supplemented this search with data from our previously published GWAS on thrombin-activatable fibrinolysis inhibitor (encoded by $C P B 2)^{25}$ and factor-seven activating protease (FSAP, encoded by HABP2). ${ }^{26}$

\section{Validation of ASM-SNPs in mQTL Data Sets}

As a way to validate our findings, ASM-SNPs and corresponding proxies $\left(r^{2} \geq 0.8\right.$ in 1,000 genomes European panel) were queried against publicly available mQTL data using PhenoScanner "Epigenetics" search function which includes data from BIOS QTL browser (https://genenetwork.nl/ biosqtlbrowser/), BLUEPRINT Epigenome (http://www.blueprint-epigenome.eu/), and Gaunt et al. ${ }^{27}$ The output result list was filtered to $p<5 \times 10^{-5}$ and manually curated to retain only $\mathrm{mQTL}$ entries.

\section{Annotation and Functional Prediction of Variants}

Database of Single Nucleotide Polymorphisms (dbSNP; build 150, available from http://www.ncbi.nlm.nih.gov/SNP/) was used to annotate SNPs. HaploReg v4.1 ${ }^{28}$ was used to determine the chromatin state in liver tissue per ASM-SNP haplotype block. The default 15-state core ChromHMM model and H3K4me1/H3K4me3 peaks from Roadmap Epigenomics 29 were used to identify enhancers and promoters. Results were manually curated to retain only variants with predicted enhancer or promoter chromatin states in liver tissue.

\section{Results}

\section{DNA Methylation Pattern and ASM of Hemostatic Genes in Human Liver}

We measured the DNA methylation status of each individual CpG in all 35 hemostatic genes including at least $5 \mathrm{~kb}$ upstream and $0.5 \mathrm{~kb}$ downstream of each gene. In total, 8,707 unique $\mathrm{CpG}$ sites were captured in our design (1.2 Mb in total). The density of $\mathrm{CpG}$ sites in each gene varied between $2 \mathrm{CpGs}($ F13B) and $30 \mathrm{CpGs}$ (F7) per kbp, with a median density of $6 \mathrm{CpGs}$ per $\mathrm{kbp}$. Schematic illustrations of DNA methylation patterns for VKORC1 (encoding vitamin $\mathrm{K}$ reductase complex subunit 1 ), $F 2$ (thrombin), and $F 10$ (factor $\mathrm{X}$ ) are displayed in - Fig. $\mathbf{1}$ and for the remaining genes in -Supplementary Fig. $\mathbf{S 2}$ (available in the online version).

Using the ASM approach, we identified 151 CpGs displaying an allelic methylation imbalance of $20 \%$ or more in at least one sample, and 116 of these were significant $(p<0.05)$. These CpGs were found in 24 of the 35 genes analyzed and were associated with 112 SNPs (-Table 1). The number of CpGs displaying ASM per gene varied between one (e.g., in VKORC1) to 15 (e.g., in SERPINF2). A schematic view of ASM positions per gene is displayed in - Fig. $\mathbf{1}$ and in - Supplementary Fig. S2 (available in the online version). The exact positions of the ASM-CpGs, the degree of ASM, dbSNP ID of the ASM-SNPs, number of heterozygous samples, and associations of ASMSNPs with mRNA expression, levels/activity of circulating proteins, and other GWAS traits with relevance for hemostasis are reported in - Tables 2-4. The ASM association results are presented in these three tables based on whether or not the ASM-SNPs have previously been identified as eQTLs. Allelic methylation for all CpGs (including nonsignificant ASM-CpGs) and further details on associations are shown in -Supplementary Table $\mathbf{5 3}$ (available in the online version). Forest plots of methylation differences between alleles in all $151 \mathrm{CpGs}$ per individual are displayed in - Supplementary Fig. $\mathbf{S 3}$ (available in the online version). A UCSC browser track for significant ASM positions is available to the research community at: https://genome.ucsc.edu/s/marcela.davila\% 40gu.se/ASM_CJern.

\section{CpGs Displaying ASM are Enriched in the First Exon}

To evaluate if ASM is overrepresented in specific genomic contexts, we performed an enrichment test comparing ASM$\mathrm{CpGs}$ with all $\mathrm{CpGs}$ in different regions. The only region with a significant over-representation of ASM was "first exon" $(p=0.0007$, Fisher's exact test).

\section{Eight ASM-SNPs Associated with mRNA Expression in RNA-Seq Data}

To determine whether DNA methylation regulated by genetic variants in cis could provide the mechanistic link between genetic variants associated with gene expression, we used mRNA-seq data (derived from the same individuals and the same liver tissue samples as used in ASM analysis) to assay potential differences in expression based on genotype of the ASM-SNP. A total of eight ASM-SNPs were significantly associated with allelic differences in expression $(p<0.05)$ : four in the gene encoding protein $\mathrm{Z}$ (PROZ), one in the gene encoding VKORC1, one in the gene encoding thrombin, and two in the gene encoding kininogen 1 (KNG1) (-Fig. 2; - Tables 2 and $\mathbf{3}$ ). A trend was observed for a further 22 ASM-SNPs ( 9 at $p<0.1$ and 13 ASM-SNPs at $p<0.2$; see online - Supplementary Fig. S4, available in the online version).

\section{Seventy-Six ASM-SNPs Associated with mRNA Expression in Publicly Available eQTL Data}

We next queried the ASM-SNPs in publicly available liver tissue eQTL data using PhenoScanner. A total of 22 ASM-SNPs (or high LD proxies) were identified as eQTLs in liver tissue for their respective gene (-Table 2 ). These included four SNPs in PROZ and one SNP in VKORC1 for which we found a significant association with mRNA expression using our RNA-seq data ( Fig. 2A-D and E, respectively) and five others with a trend for association. The same alleles associated with higher gene expression in liver tissue eQTL studies were expressed at higher levels in our RNA-seq data (data not shown).

While many cis-acting genetic variants are specific for a given tissue, others may be common to several tissues. ${ }^{22}$ 

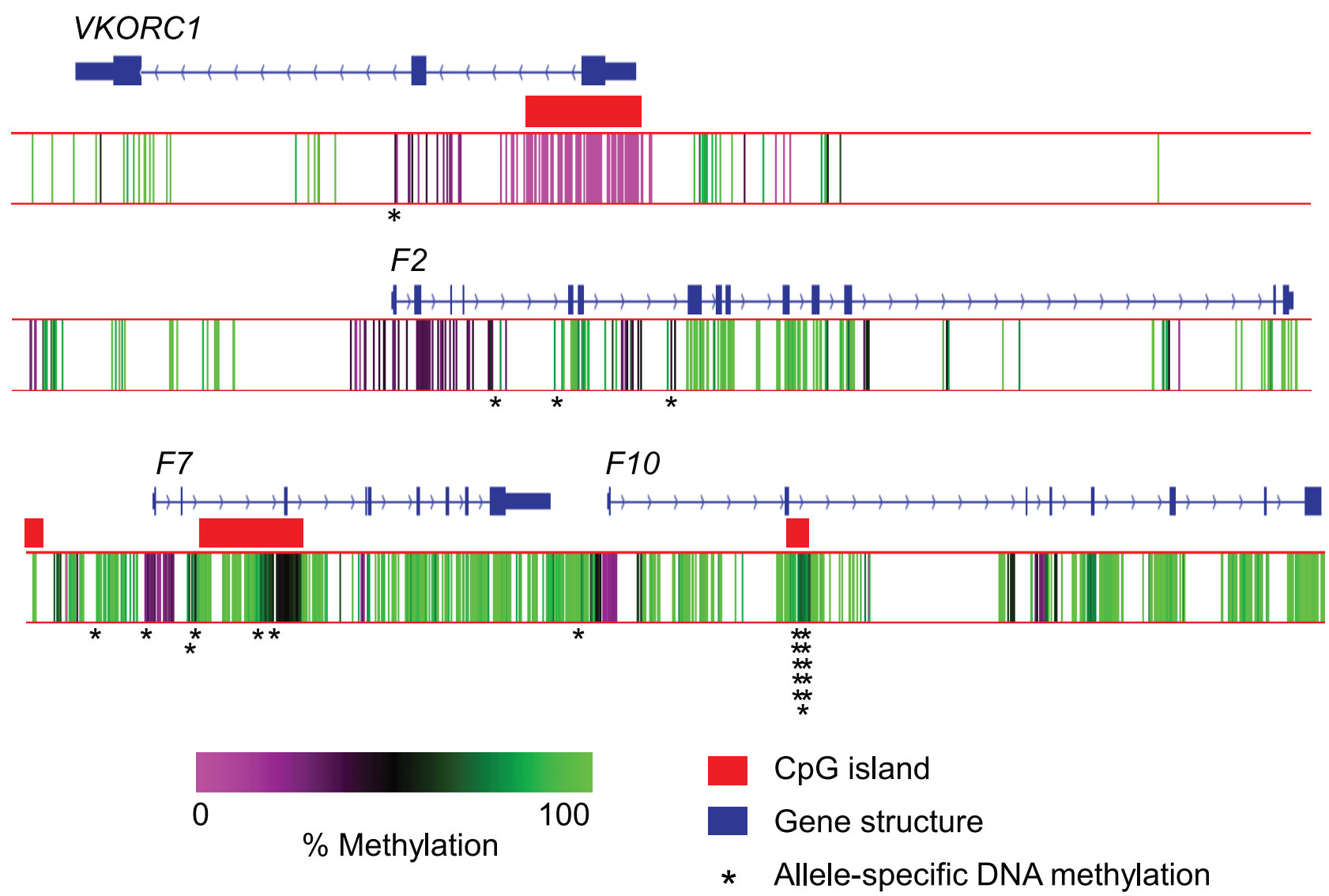

Fig. 1 Deoxyribonucleic acid (DNA) methylation patterns of selected hemostatic genes in human liver tissue. For each gene, gene structure (blue) and cytosine-guanine dinucleotide (CpG) islands (red) are displayed on top. At least $5 \mathrm{~kb}$ upstream and $0.5 \mathrm{~kb}$ downstream of each gene are included. The methylation status for all $\mathrm{CpGs}$ is displayed, where purple represents a low degree of methylation and green a high degree of methylation. Methylation values are based on the average of the 19 samples. CpGs displaying allele-specific methylation (ASM) in one or more samples are denoted by asterisks. (A) VKORC1; (B) F2; and (C) F7 and F10. All other genes can be found in - Supplementary Fig. $\mathbf{2 2}$ (available in the online version).

Further, sample size greatly affects eQTL mapping and thus more readily available tissues tend to have more reported eQTLs. $^{22}$ In line with this, an additional 54 ASM-SNPs (or proxies) were eQTLs for their respective genes in other tissues in a total of 17 genes ( - Table 3 ). This included four SNPs in $P R O Z$, one in $F 2$, and two in KNG1, significantly associated with expression using our normalized RNA-seq data (-Fig. 2F-H), and 13 other SNPs showing a trend for association in our data ( - Supplementary Fig. S4, available in the online version). A complete list of all eQTL associations from all tissues can be found in - Supplementary Table S4 (available in the online version).

For 36 ASM-SNPs (corresponding to 16 genes), no genotype-expression associations have previously been reported (-Table 4). Of these, three ASM-SNPs exhibited a trend for association with expression using our normalized RNA-seq data for $P R O Z$ and the genes encoding factor V (F5) and $\alpha-2-$ plasmin inhibitor (SERPINF2).

\section{Thirty-Three ASM-SNPs Associate with Circulating Proteins; 36 with Other Relevant GWAS Traits}

To determine whether allele-specific DNA methylation also could affect protein abundance, we assessed ASM-SNPs for association with circulating levels or activity of plasma proteins using publicly available protein quantitative trait loci (pQTL) and GWAS data sets. A total of 33 ASM-SNPs (or proxies) were previously associated with the respective protein concentration or activity (-Tables 2-4, details in -Supplementary Table S3, available in the online version), and these included 5 ASM-SNPs with no previous reported eQTLs that were associated with plasma fibrinogen, KNG1, SERPINF2, and FSAP levels ( - Table 4). A complete list of pQTL associations can be found in -Supplementary Table 55 (available in the online version).

We next assessed association of ASM-SNPs with relevant disease and nondisease phenotypes in PhenoScanner. One ASM-SNP in VKORC1 was associated with warfarin dosage levels (rs8050894). ${ }^{30}$ A total of four ASM-SNPs were associated with complex diseases (two SNPs in F5 with venous thromboembolism [rs3766110, rs3766111; http://www.ukbiobank.ac. uk], one SNP in TFPI with coronary artery disease [rs8176546], ${ }^{31}$ and one in F2 with stroke [rs2070851] ${ }^{32}$ ). A total of 33 ASM-SNPs were associated with other related phenotypes. A complete list of GWAS associations can be found in -Supplementary Table S5 (available in the online version). 


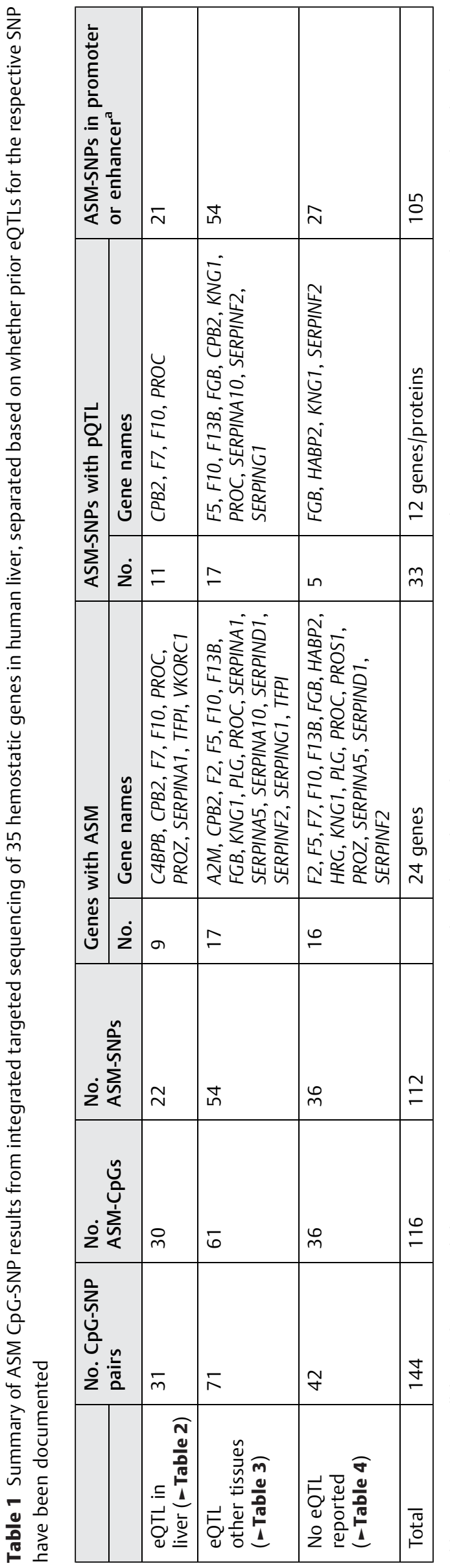

\section{Overlap between ASM-SNPs and Previously Identified MQTL SNPS}

We queried our ASM-CpGs against the publicly available mQTL microarray-based data using PhenoScanner. Only six of the CpGs displaying ASM in our study design are located on commercially available microarrays. However, all six of these were identified as mQTLs with the same SNP-CpG association as in our study, although in other tissue types than liver. Therefore, all remaining ASM-CpGs identified here are completely novel genotype-associated methylation sites, and all associations reported in this study are novel in liver tissue.

\section{Discussion}

In this study, we analyzed DNA methylation profiles of 35 hemostatic genes that are predominantly expressed in liver. To the best of our knowledge, this is the first study presenting DNA methylation patterns of hemostatic genes on an individual CpG level in human liver. We identified ASM in 24 of the 35 targeted genes. In addition, we demonstrated associations between ASM-SNPs and mRNA expression as well as association with circulating hemostatic proteins, relevant complex traits (e.g., coronary artery disease, stroke, venous thromboembolism), and drug dosing (e.g., warfarin). Our findings highlight the importance of genetic-epigenetic interactions and suggest that methylation regulated by genetic variants could provide the mechanism by which SNPs exert an effect on circulating hemostatic proteins, prothrombotic diseases, and drug response.

As discussed in the Introduction, previous studies that link variations in genotypes to DNA methylation levels at specific CPG sites across individuals (mQTL) require large sample sizes and have most often been performed in easy to access tissues such as blood. Here, we used ASM analysis, which has an advantage over MQTL analyses as it uses a within-sample control (the other allele) to query effects of SNPs on DNA methylation. In doing so, the ASM approach is more robust to environmental and experimental effects and thus more sensitive than standard mQTL analysis. ${ }^{33}$ We were therefore able to use human liver tissue, the main biological source of the hemostatic proteins studied here, to map putative cis-acting polymorphisms despite having a small number of samples. ${ }^{17}$ ASM-SNPs and corresponding proxies were queried against publicly available MQTL data as a way to validate our findings. However, as nearly all published human mQTL studies have used commercially available microarrays that interrogate only a small portion of all CpGs, only eight CpGs in our study design are represented in MQTL data. Six of these displayed ASM in our data, and all of these were identified as mQTLs with the same SNP-CpG association as in our study, although in other tissue types than liver. Ideally, we would compare our ASM results with another similar bisulfite-seq data set in liver, but such data is not available at this time as far as we are aware.

The hemostatic genes analyzed in this study all had a low degree of methylation around promoters, as expected for actively transcribed genes (-Fig. 1 and 
Table 2 CpGs displaying significant ASM $(p<0.05)$ and associated SNPs (ASM-SNPs) previously reported as liver eQTLs in public databases, and associations of the ASM-SNPs to mRNA expression in liver tissue, to circulating protein levels of the respective genes, and to other GWAS traits relevant for hemostasis

\begin{tabular}{|c|c|c|c|c|c|c|c|c|}
\hline & ASM & & & SNP & & Associa & ons & \\
\hline Gene & CpG location & No. het. & \% ASM diff. & $\mathrm{dbSNP}$ & $\begin{array}{l}\text { Distance from } \\
\text { CpG (bp) }\end{array}$ & mRNA & $\mathrm{pQTL}$ & GWAS \\
\hline$C 4 B P B$ & chr1:207269856 & 1 & -21 & rs8942 & 63 & Yes $^{\mathrm{b}}$ & & \\
\hline CPB2 & chr13:46644103 & 2 & 9 & rs9534307 & 27 & Yes $^{\mathrm{b}}$ & Yes & \\
\hline CPB2 & chr13:46649378 & 1 & -27 & rs9567613 & 20 & Yes $^{\mathrm{b}}$ & Yes & \\
\hline CPB2 & chr13:46669605 & 1 & -21 & rs7991899 & -55 & Yes $^{\mathrm{b}}$ & Yes & \\
\hline CPB2 & chr13:46683614 & 2 & -10 & rs7338061 & 19 & Yes $^{\mathrm{b}}$ & Yes & \\
\hline$F 10$ & chr13:113784439a & 1 & 23 & rs693335 & 4 & & Yes & Yes \\
\hline$F 10$ & chr13:113784452 & 1 & 20 & rs693335 & -9 & & Yes & Yes \\
\hline$F 10$ & chr13:113784460 & 1 & 25 & rs693335 & -17 & & Yes & Yes \\
\hline F10 & chr13:113784466 & 1 & 26 & rs693335 & -23 & & Yes & Yes \\
\hline$F 10$ & chr13:113784481 & 1 & 22 & rs693335 & -38 & & Yes & Yes \\
\hline F10 & chr13:113784497 & 1 & 23 & rs693335 & -54 & & Yes & Yes \\
\hline$F 10$ & chr13:113784499 & 1 & 25 & rs693335 & -56 & & Yes & Yes \\
\hline$F 7$ & chr13:113757759 & 2 & -12 & rs3093229 & 67 & & Yes & Yes \\
\hline F7 & chr13:113759695 & 1 & -25 & rs510317 & 59 & & Yes & Yes \\
\hline F7 & chr13:113761451 & 2 & 24 & rs28663357 & 62 & Yes $^{\mathrm{b}}$ & & Yes \\
\hline F7 & chr13:113761585 & 2 & 15 & rs142493427 & 5 & & & Yes \\
\hline PROC & chr2:128174852 & 1 & -28 & rs2069901 & -9 & & Yes & \\
\hline PROC & chr2:128174852 & 1 & -28 & rs2069902 & 11 & & Yes & \\
\hline PROC & chr2:128176028 & 9 & -6 & rs1799810 & 12 & & Yes & \\
\hline PROC & chr2:128177360 & 8 & 11 & rs1158867 & 17 & & Yes & \\
\hline PROZ & chr13:113817559 & 1 & 45 & rs2480948 & -22 & Yes & $\mathrm{NA}$ & Yes \\
\hline PROZ & chr13:113818668 & 2 & -24 & rs3024731 & 40 & Yes & $\mathrm{NA}$ & Yes \\
\hline PROZ & chr13:113819002 & 5 & 26 & rs494860 & 7 & Yes & $\mathrm{NA}$ & Yes \\
\hline PROZ & chr13:113819008 & 5 & 27 & rs494860 & 1 & Yes & $\mathrm{NA}$ & Yes \\
\hline PROZ & chr13:113819050 & 2 & 29 & rs494860 & -41 & Yes & NA & Yes \\
\hline PROZ & chr13:113819074 & 2 & 18 & rs494860 & -65 & Yes & $\mathrm{NA}$ & Yes \\
\hline PROZ & chr13:113824222 & 1 & 32 & rs1755690 & 44 & Yes & NA & Yes \\
\hline SERPINA1 & chr14:94844610 & 1 & -24 & rs2073333 & -48 & & NA & \\
\hline SERPINA1 & chr14:94844885 & 1 & 31 & rs1303 & -42 & & $\mathrm{NA}$ & \\
\hline TFPI & chr2:188340376 & 4 & -11 & rs8176546 & 20 & & NA & Yes \\
\hline VKORC1 & chr16:31104505 & 3 & -47 & rs8050894 & 4 & Yes & NA & Yes \\
\hline
\end{tabular}

Abbreviations: ASM, allele-specific DNA methylation; bp, base pairs; CpG, cytosine-guanine site; dbSNP, Database of Single Nucleotide Polymorphisms; DNA, deoxyribonucleic acid; eQTL, expression quantitative trait loci; GWAS, genome-wide association study; mRNA, messenger ribonucleic acid; $\mathrm{PQTL}$, protein quantitative trait loci; SNP, single-nucleotide polymorphism.

Note: No. het., number of heterozygous samples; \% ASM diff., mean percent methylation difference between major and minor alleles of all heterozygous samples. A positive percent difference indicates higher methylation level on the major allele.

${ }^{a} \mathrm{CpG}$ included on commercial DNA methylation microarrays.

bonsignificant $(p>0.05)$ trend for association with mRNA expression in in-house mRNA-seq data.

-Supplementary Fig. S2, available in the online version). The methylation level in gene bodies was generally much higher, although this varied more than the promoter methylation ( Fig. 1 and - Supplementary Fig. S2, available in the online version). Previous mQTL studies in a variety of tissues have demonstrated that cis-acting methylation-associated SNPs are enriched in regulatory chromatin domains and transcription factor binding sites, and colocalize with eQTLs. ${ }^{11,34-37}$ Further, CpG sites that are associated with expression levels have previously been shown to be enriched in enhancers, 
236 Allele-Specific Methylation of Hemostatic Genes Olsson Lindvall et al.

Table 3 CpGs displaying significant ASM $(p<0.05)$ and associated SNPs (ASM-SNPs) previously reported as eQTLs in tissue types other than liver, and association of these ASM-SNPs to mRNA expression in liver tissue (RNA-seq data), to circulating protein levels of the respective genes ( $\mathrm{PQTL}$ ), and to other GWAS traits relevant for hemostasis

\begin{tabular}{|c|c|c|c|c|c|c|c|c|}
\hline \multirow[b]{2}{*}{ Gene } & \multicolumn{3}{|l|}{ ASM } & \multicolumn{2}{|l|}{ SNP } & \multicolumn{3}{|c|}{ Associations } \\
\hline & CpG location & No. het. & \% ASM diff. & dbSNP & $\begin{array}{l}\text { Distance from } \\
\text { CpG (bp) }\end{array}$ & mRNA & pQTL & GWAS \\
\hline$A 2 M$ & chr12:9230027 & 7 & -18 & rs2377682 & 11 & & NA & \\
\hline$A 2 M$ & chr12:9259915 & 6 & -10 & rs226389 & -8 & $Y_{e s}^{b}$ & NA & \\
\hline$A 2 M$ & chr12:9279617 & 7 & -6 & rs226372 & -19 & $Y_{e s}^{b}$ & NA & \\
\hline$C P B 2$ & chr13:46627428 & 8 & -28 & rs1087 & 11 & Yes $^{\mathrm{b}}$ & Yes & \\
\hline$C P B 2$ & chr13:46627428 & 8 & -28 & rs940 & 52 & & Yes & \\
\hline$C P B 2$ & chr13:46627473 & 4 & -23 & rs940 & 7 & & Yes & \\
\hline CPB2 & chr13:46627504 & 3 & -12 & rs940 & -24 & & Yes & \\
\hline CPB2 & chr13:46649378 & 1 & -27 & rs1022952 & -30 & & Yes & \\
\hline F10 & chr13:113784564 & 1 & 36 & rs776906 & 24 & & Yes & Yes \\
\hline F10 & chr13:113784692 & 1 & 21 & rs2480946 & 28 & & Yes & Yes \\
\hline F10 & chr13:113784702 & 1 & -24 & rs2480946 & 18 & & Yes & Yes \\
\hline F10 & chr13:113776916 a & 4 & -11 & rs563964 & 31 & Yes $^{\mathrm{b}}$ & & Yes \\
\hline F10 & chr13:113776916 & 4 & -11 & rs3211717 & 33 & Yes $^{\mathrm{b}}$ & Yes & \\
\hline$F 13 B$ & chr1:197026831 & 10 & 8 & rs17549312 & 15 & & Yes & \\
\hline$F 13 B$ & chr1:197026871 & 6 & 12 & rs17549312 & -25 & & Yes & \\
\hline$F 2$ & chr11:46744514 & 2 & 17 & rs2070851 & 14 & & NA & Yes \\
\hline$F 2$ & chr11:46747073 & 1 & -50 & rs3136460 & -8 & Yes & NA & Yes \\
\hline F5 & chr1:169493712 & 1 & 25 & rs9332641 & 1 & & Yes & Yes \\
\hline F5 & chr1:169493712 & 1 & 25 & rs9332640 & 14 & & Yes & \\
\hline F5 & chr1:169515148 & 1 & -27 & rs3766110 & 35 & & & Yes \\
\hline F5 & chr1:169515148 & 1 & -27 & rs3766111 & 56 & & & Yes \\
\hline F5 & chr1:169533986 & 1 & 21 & rs724507 & 42 & & & \\
\hline F5 & chr1:169555972 & 1 & -25 & rs2269648 & 78 & & & \\
\hline$F G B$ & chr4:155486657 & 3 & 12 & rs2227403 & -11 & & Yes & \\
\hline KNG1 & chr3:186435526 & 1 & -22 & rs13072823 & 35 & Yes & & Yes \\
\hline KNG1 & chr3:186440466 & 2 & 16 & rs1648714 & 17 & & Yes & Yes \\
\hline KNG1 & chr3:186449900 & 6 & -6 & rs1622922 & -16 & Yes $^{\mathrm{b}}$ & & Yes \\
\hline KNG1 & chr3:186449900 & 6 & -6 & rs1648699 & -10 & Yes $^{\mathrm{b}}$ & & Yes \\
\hline KNG1 & chr3:186449900 & 6 & -6 & rs1648700 & 16 & Yes $^{\mathrm{b}}$ & & Yes \\
\hline KNG1 & chr3:186452968 & 2 & -19 & rs822363 & 23 & & & Yes \\
\hline KNG1 & chr3:186434963 & 2 & -16 & rs5029973 & -76 & Yes & & Yes \\
\hline$P L G$ & chr6:161135056 & 2 & 11 & rs3757019 & 55 & Yes $^{\mathrm{b}}$ & & \\
\hline$P L G$ & chr6:161137753 & 2 & 16 & rs14224 & 26 & Yes $^{\mathrm{b}}$ & & \\
\hline PROC & chr2:128182990 & 8 & 29 & rs2069924 & 4 & & Yes & \\
\hline PROC & chr2:128147838 & 7 & 8 & rs13029237 & 16 & & Yes & \\
\hline PROC & chr2:128148742 & 6 & -22 & rs35708549 & 22 & & & \\
\hline PROC & chr2:128148776 & 8 & -27 & rs35708549 & -12 & & & \\
\hline SERPINA1 & chr14:94843725 & 1 & 23 & rs11628917 & -6 & & NA & Yes \\
\hline SERPINA1 & chr14:94852549 & 1 & 25 & rs1980617 & -15 & & NA & Yes \\
\hline SERPINA1 & chr14:94852749 & 2 & -16 & rs75285293 & 47 & & NA & \\
\hline
\end{tabular}


Table 3 (Continued)

\begin{tabular}{|c|c|c|c|c|c|c|c|c|}
\hline \multirow[b]{2}{*}{ Gene } & \multicolumn{3}{|l|}{ ASM } & \multicolumn{2}{|l|}{ SNP } & \multicolumn{3}{|c|}{ Associations } \\
\hline & CpG location & No. het. & \% ASM diff. & dbSNP & $\begin{array}{l}\text { Distance from } \\
\text { CpG (bp) }\end{array}$ & mRNA & pQTL & GWAS \\
\hline SERPINA1 & chr14:94858368 & 2 & -12 & rs1610317 & -12 & & NA & Yes \\
\hline SERPINA10 & chr14:94755152 & 2 & -22 & rs12434093 & 32 & & Yes & \\
\hline SERPINA10 & chr14:94755217 & 1 & -21 & rs12434093 & -33 & & Yes & \\
\hline SERPINA10 & chr14:94755230 & 1 & -32 & rs12434093 & -46 & & Yes & \\
\hline SERPINA10 & chr14:94760975 & 5 & -9 & rs3827897 & -10 & & Yes & \\
\hline SERPINA5 & chr14:95050296 & 2 & -22 & rs12880862 & 12 & & & \\
\hline SERPINA5 & chr14:95051504 & 1 & -40 & rs12886695 & -64 & & & \\
\hline SERPINA5 & chr14:95047617 & 2 & -12 & rs11847476 & -12 & Yes $^{\mathrm{b}}$ & & \\
\hline SERPINA5 & chr14:95047617 & 2 & -12 & rs11847477 & -9 & & & \\
\hline SERPIND1 & chr22:21129590 & 1 & 25 & rs165680 & -31 & Yes $^{\mathrm{b}}$ & NA & \\
\hline SERPINF2 & chr17:1648204 & 1 & -32 & rs2070862 & 90 & & & \\
\hline SERPINF2 & chr17:1650172 & 1 & -40 & rs117378188 & 31 & Yes $^{\mathrm{b}}$ & & \\
\hline SERPINF2 & chr17:1658948 & 1 & -23 & rs4566204 & 20 & & & \\
\hline SERPINF2 & chr17:1658955 & 1 & -26 & rs4566204 & 13 & & & \\
\hline SERPINF2 & chr17:1636455 & 1 & -29 & rs11657222 & 22 & Yes $^{\mathrm{b}}$ & & \\
\hline SERPINF2 & chr17:1640652 & 1 & 46 & rs62090051 & -12 & & & \\
\hline SERPINF2 & chr17:1640661 & 1 & 36 & rs62090051 & -21 & & & \\
\hline SERPINF2 & chr17:1640674 & 1 & 44 & rs62090051 & -34 & & & \\
\hline SERPINF2 & chr17:1640674 & 1 & 44 & rs1045794 & 35 & Yes $^{b}$ & & \\
\hline SERPINF2 & chr17:1640710 & 1 & -30 & rs62090051 & -70 & & & \\
\hline SERPINF2 & chr17:1640710 & 1 & -30 & rs1045794 & -1 & Yes $^{\mathrm{b}}$ & & \\
\hline SERPINF2 & chr17:1640788 & 11 & -7 & rs8077638 & 5 & & Yes & \\
\hline SERPINF2 & chr17:1640794 & 1 & -31 & rs1045794 & -85 & Yes $^{b}$ & & \\
\hline SERPINF2 & chr17:1640794 & 1 & -31 & rs8077638 & -1 & & Yes & \\
\hline SERPINF2 & chr17:1640812 & 10 & -10 & rs8077638 & -19 & & Yes & \\
\hline SERPINF2 & chr17:1640830 & 4 & -18 & rs8077638 & -37 & & Yes & \\
\hline SERPINF2 & chr17:1640868 & 1 & -21 & rs 8077638 & -75 & & Yes & \\
\hline SERPINF2 & chr17:1645507 & 5 & -14 & rs62090057 & 19 & & & \\
\hline SERPING1 & chr11:57374280 & 1 & -22 & rs11603020 & 52 & Yes $^{\mathrm{b}}$ & Yes & \\
\hline TFPI & chr2:188340376 & 4 & -11 & rs8176547 & -27 & & NA & \\
\hline TFPI & chr2:188353789 & 1 & 36 & rs 8176500 & -35 & & NA & \\
\hline
\end{tabular}

Abbreviations: ASM, allele-specific DNA methylation; bp, base pairs; CpG, cytosine-guanine site; dbSNP, Database of Single Nucleotide Polymorphisms; DNA, deoxyribonucleic acid; eQTL, expression quantitative trait loci; GWAS, genome-wide association study; mRNA, messenger ribonucleic acid; $\mathrm{PQTL}$, protein quantitative trait loci; SNP, single-nucleotide polymorphism.

Note: No het, number of heterozygous samples; \% ASM diff., mean percent methylation difference between major and minor alleles of all heterozygous samples. A positive percent difference indicates higher methylation level on the major allele.

${ }^{a} \mathrm{CpG}$ included on commercial DNA methylation microarrays.

${ }^{\mathrm{b}}$ Nonsignificant $(p>0.05)$ trend for association with mRNA expression in in-house RNA-seq data.

gene bodies, $\mathrm{CpG}$ island shores, and in the first exon, but not in promoter regions. ${ }^{8,38}$ In line with this, we found that the majority of the ASM-CpGs/SNPs were located within gene bodies (i.e., 102 out of 144) and identified an enrichment of ASM in the first exon. Additionally, nearly all (94\%) of the ASM-SNPs had chromatin states indicative of promoter and/or enhancer elements in liver (- Supplementary Table 56, available in the online version). Taken together, we present the first detailed map of the DNA methylation landscape in hemostatic genes in human liver tissue, and our study underscores the importance of more mechanistic studies on the role of methylation in gene bodies. 
238 Allele-Specific Methylation of Hemostatic Genes Olsson Lindvall et al.

Table 4 CpGs displaying significant ASM $(p<0.05)$ and associated SNPs (ASM-SNPs) not reported as eQTLs in public databases, and associations of ASM-SNPs to mRNA expression in liver tissue (RNA-seq data), to circulating protein levels of the respective genes (pQTL), and to other GWAS traits relevant for hemostasis

\begin{tabular}{|c|c|c|c|c|c|c|c|c|}
\hline \multirow[b]{2}{*}{ Gene } & \multicolumn{3}{|l|}{ ASM } & \multicolumn{2}{|l|}{ SNP } & \multicolumn{3}{|c|}{ Associations } \\
\hline & CpG location & No. het. & $\%$ ASM diff. & $\mathrm{dbSNP}$ & Distance from CpG (bp) & mRNA & PQTL & GWAS \\
\hline F10 & chr13:113784664 & 1 & 31 & rs3211742 & -20 & & & \\
\hline$F 13 B$ & chr1:197026871 & 6 & 12 & rs1759008 & 24 & & & Yes \\
\hline$F 2$ & chr11:46743111 & 4 & 17 & rs3136439 & 24 & & NA & \\
\hline$F 5$ & chr1:169515447 & 1 & 42 & rs749045929 & -29 & & & \\
\hline F5 & chr1:169515447 & 1 & 42 & rs3835453 & -25 & & & \\
\hline$F 5$ & chr1:169515447 & 1 & 42 & rs796545997 & -27 & Yes $^{\mathrm{b}}$ & & Yes \\
\hline$F 5$ & chr1:169515447 & 1 & 42 & rs112177003 & -1 & & & \\
\hline$F 5$ & chr1:169529841 & 1 & 24 & rs6022 & -15 & & & \\
\hline$F 7$ & chr13:113759695 & 1 & -25 & rs510335 & 60 & & & \\
\hline$F 7$ & chr13:113761585a & 2 & 15 & rs2515646 & 17 & & & \\
\hline$F G B$ & chr4:155480425 & 1 & 21 & rs7673587 & 9 & & Yes & \\
\hline HABP2 & chr10:115310488 & 5 & -9 & rs2000277 & 26 & & & \\
\hline HABP2 & chr10:115310548 & 6 & 12 & rs3758591 & 4 & & Yes & \\
\hline HABP2 & chr10:115310548 & 6 & 12 & rs3758590 & 15 & & & \\
\hline HABP2 & chr10:115316814 & 1 & 32 & rs4918842 & -2 & & & \\
\hline HABP2 & chr10:115327006 & 1 & 23 & rs11575665 & 34 & & & \\
\hline HABP2 & chr10:115327006 & 1 & 23 & rs11575666 & 42 & & & \\
\hline HABP2 & chr10:115330374 & 7 & 56 & rs7079148 & 12 & & & \\
\hline HABP2 & chr10:115332849 & 10 & 33 & rs7101144 & 1 & & & \\
\hline HABP2 & chr10:115334080 & 2 & 13 & rs3740530 & 44 & & Yes & \\
\hline HABP2 & chr10:115336796 & 1 & 24 & rs2302374 & -38 & & & \\
\hline$H R G$ & chr3:186383214 & 1 & -21 & rs9814412 & -34 & & & Yes \\
\hline KNG1 & chr3:186442126 & 1 & -20 & rs 1648712 & 83 & & & Yes \\
\hline KNG1 & chr3:186445048 & 2 & 16 & rs2304456 & 4 & & Yes & Yes \\
\hline KNG1 & chr3:186445221 & 2 & 30 & rs3841557 & -35 & & & \\
\hline KNG1 & chr3:186445221 & 2 & 30 & rs5030025 & 16 & & & Yes \\
\hline$P L G$ & chr6:161126010 & 1 & -22 & rs1819138 & 64 & & & \\
\hline$P L G$ & chr6:161126030 & 1 & -35 & rs1819138 & 44 & & & \\
\hline$P L G$ & chr6:161168694 & 1 & -27 & rs10625439 & -41 & & & \\
\hline PROC & chr2:128148742 & 6 & -22 & rs79819102 & -1 & & & \\
\hline PROC & chr2:128148776 & 8 & -27 & rs79819102 & -35 & & & \\
\hline PROS1 & chr3:93605725 & 1 & -45 & rs8178648 & 14 & & $\mathrm{NA}$ & \\
\hline PROZ & chr13:113817557 & 1 & 49 & rs45538837 & -2 & & NA & Yes \\
\hline PROZ & chr13:113817559 & 1 & 45 & rs45538837 & -4 & & NA & Yes \\
\hline PROZ & chr13:113818958 & 2 & 16 & rs3024733 & 44 & & NA & \\
\hline PROZ & chr13:113819002 & 5 & 26 & rs3024733 & 0 & & NA & \\
\hline PROZ & chr13:113819008 & 5 & 27 & rs3024733 & -6 & & NA & \\
\hline PROZ & chr13:113819093 & 1 & 23 & rs3024782 & 65 & Yes $^{\mathrm{b}}$ & $\mathrm{NA}$ & \\
\hline PROZ & chr13:113819101 & 1 & 25 & rs3024782 & 57 & Yes $^{\mathrm{b}}$ & NA & \\
\hline SERPINA5 & chr14:95048706 & 1 & -27 & rs112795992 & -1 & & & \\
\hline SERPIND1 & chr22:21132447 & 2 & -32 & rs75917143 & -9 & & $\mathrm{NA}$ & \\
\hline SERPINF2 & chr17:1650172 & 1 & -40 & rs6502935 & -4 & Yes $^{\mathrm{b}}$ & Yes & \\
\hline
\end{tabular}

Abbreviations: ASM, allele-specific DNA methylation; bp, base pairs; CpG, cytosine-guanine site; dbSNP, Database of Single Nucleotide Polymorphisms; DNA, deoxyribonucleic acid; eQTL, expression quantitative trait loci; GWAS, genome-wide association study; mRNA, messenger ribonucleic acid; PQTL, protein quantitative trait loci; SNP, single-nucleotide polymorphism.

Note: No. het., number of heterozygous samples; \% ASM diff., mean percent methylation difference between major and minor alleles of all heterozygous samples. A positive percent difference indicates higher methylation level on the major allele.

${ }^{\mathrm{a}} \mathrm{CpG}$ included on commercial DNA methylation microarrays.

${ }^{\mathrm{b}}$ Nonsignificant $(p>0.05)$ trend for association with mRNA expression in in-house RNA-seq data. 

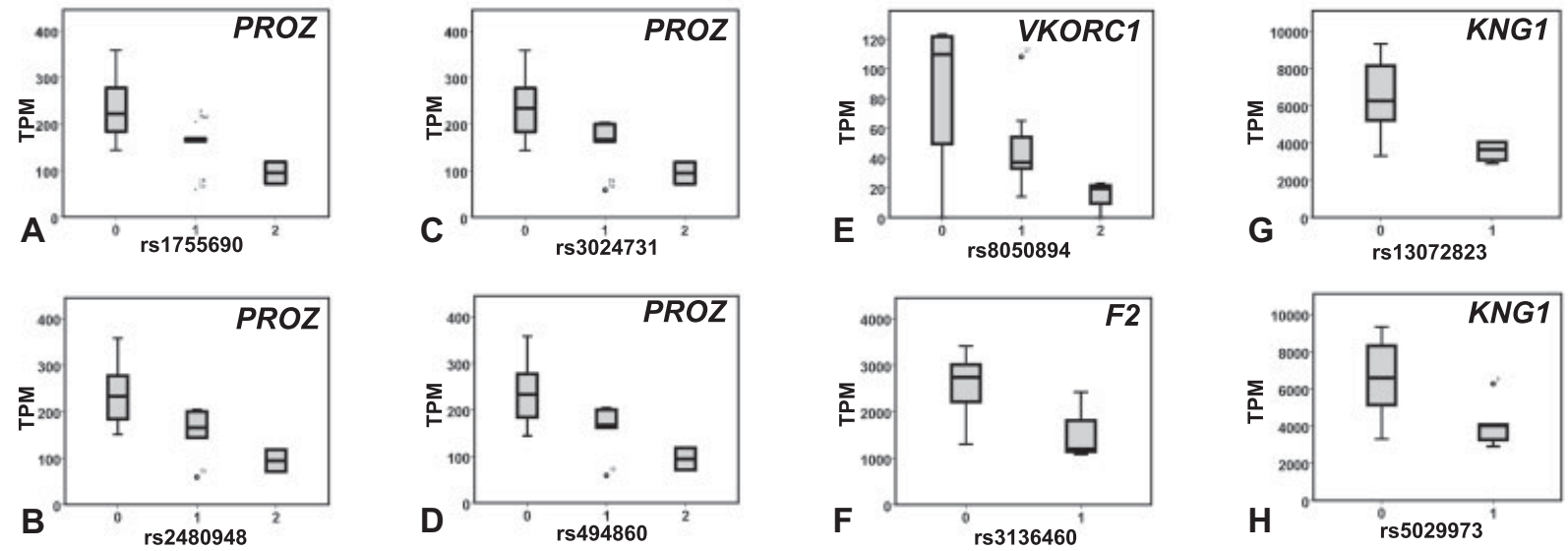

Fig. 2 Box plots of genotype versus messenger ribonucleic acid (mRNA) expression level. Allele-specific methylation-single-nucleotide polymorphisms (ASM-SNPs) significantly associated to expression of the respective gene based on normalized RNA-seq data from 19 human liver samples $(p<0.05)$. Subjects homozygous for the major allele had higher gene expression of PROZ (A-D); VKORC1 (E); F2 (F); and KNG1 (G, H). TPM, transcripts per million. 0 = homozygous major allele, $1=$ heterozygous, $2=$ homozygous minor allele. Plots for ASM-SNPs showing a trend for association to expression $(p<0.2)$ can be found in - Supplementary Fig. S2 (available in the online version).

We found that $68 \%$ of our ASM-SNPs had previously been associated with expression of their respective genes in publicly available eQTL databases. However, only a minority (22 of 76) were liver eQTLs. Thus, the ASM-SNPs were also tested for association to expression using RNA-seq data from our liver samples. We report both significant associations and trends. We are aware that such an analysis should ideally be adjusted for multiple testing. However, as we were underpowered for inter-individual analysis, we believe this is an appropriate first step as we used the tissue of relevance. ${ }^{39}$ In addition to coming from the same individuals, DNA methylation and RNA analyses were analyzed from the same tissue samples (i.e., DNA and RNA were extracted simultaneously, with the same exact cell composition) in our study which can facilitate the investigation of the functional relationship between DNA methylation and gene expression. ${ }^{40}$ In support of our RNA-seq results, all significant associations were previously reported eQTLs (five in liver and three in other tissues). Of the 22 ASM-SNPs with a trend for association, 19 were previously reported eQTLs (6 in liver and 13 in other tissues). Therefore, despite using so few samples, our study supports many published eQTLs for hemostatic genes, even those initially identified in other tissues, and provides validity to our study design.

eQTLs tend to be found in promoter regions, whereas mQTLs and cis-ASM positions tend to occur in other regulatory regions such as enhancers and insulators. ${ }^{33}$ Previous studies have found only a small overlap between variants tagging cis-acting DNA methylation and expression (i.e., mQTLs and eQTLs). ${ }^{11,15,27,41-43}$ We recently performed a genotype-expression study by analyzing allele-specific expression (ASE) of the same 35 hemostatic genes in the same sample used in the present study. ${ }^{18}$ We compared all ASMSNPs and ASE-SNPs (including corresponding proxies) identified in both studies and found only 10 SNPs in common $(<1 \%)$. As ASE analyses are limited to exonic SNPs (i.e., SNPs need to be present on RNA), whereas ASM is based on CpG sites that are located primarily in upstream regulatory regions and introns, these two analyses represent complementary approaches to identify novel cis-regulatory variants. Taken together, the results from our previous and present study highlight the complimentary nature of conducting both types of mapping.

Given the range of processes that affect protein abundance, a SNP influencing DNA methylation and mRNA levels may not necessarily influence the protein level. To address whether DNA methylation regulated by SNPs in cis could provide the mechanistic link between SNPs previously associated with circulating hemostatic proteins, we intersected the ASM-SNPs (or high LD proxies) with public PQTL and GWAS data. As far as we are aware, pQTL or GWAS protein data (for SNPs in the respective gene) is only available for 16 of the 24 genes displaying ASM in our study. For 12 of these genes (75\%), the SNPs associated with protein levels in the public data were the same as the ASM-SNPs (associated with CpG methylation) identified here. Although this analysis suggests a potential role for many of the ASM-SNPs in regulation of plasma proteins, it should be emphasized that further replication of our results and other functional validation studies are warranted.

In the era of personalized therapy, several clinical trials are investigating the value of genotype-guided dosing of the most commonly used oral anticoagulants, for example, warfarin, and nonvitamin $\mathrm{K}$ antagonist oral anticoagulants (NOACs). ${ }^{44-46}$ Studies are also emerging that demonstrate that DNA methylation changes contribute to the variability of response to warfarin, ${ }^{47}$ aspirin, and clopidogrel. ${ }^{48}$ Interestingly and in line with this, we identified ASM CpG-SNP pairs in VKORC1 which is a target for warfarin, and in F2 and F10 which both are targets for NOACs. We also identified ASM CpG-SNP pairs in 11 other hemostatic genes that are current targets of approved antithrombotic, antifibrinolytic, and other related drugs. ${ }^{49}$ The ASM-SNP in VKORC1 is an eQTL 
SNP in liver for VKORC1 expression, was associated with mRNA expression in our study ( $p=0.009$ ), and is the top hit in a GWAS for "warfarin maintenance dose." ${ }^{30}$ The ASM-CpG associated with this SNP displayed one of the highest degrees of ASM in our study, with a percent methylation difference of $47 \%$ in favor of the minor allele. A SNP in almost perfect LD with the ASM-SNP has already been used in pharmacogenetics-based clinical trials for warfarin with promising results in European populations. ${ }^{44}$ Thus, methylation influenced by genetic variation in cis may not only provide a mechanistic link between the noncoding genetic changes and phenotypic variation observed in circulating hemostatic proteins and prothrombotic diseases but also in response to drugs.

Apart from the methodological strengths of the ASM approach described above, we used $\mathrm{N}$-masked individualized genomes based on gDNA-seq data to prevent alignment bias which is otherwise a common source of error in ASM analysis. Additionally, the custom target sequence design used here provides much deeper coverage across the genes of interest compared with whole genome bisulfite-seq. Despite these strengths, there are some limitations which deserve mention. (1) The targeted design we chose was limited to approximately $5 \mathrm{~kb}$ upstream and $0.5 \mathrm{~kb}$ downstream of each gene, which means we could have missed important SNPs or CpGs outside of these regions. However, it is of note that our design still provides a great enrichment compared with commonly used array-based analyses. (2) Although the ASM approach is powerful, our study is based on just 19 samples and increasing the number of samples may reveal many more CpG-SNP associations. (3) We used conventional (short-read) Illumina sequencing. To assess for ASM, one must obtain both allelic and methylation signals on the same sequencing read. Therefore, using a platform that produces longer reads (e.g., single-molecule real-time bisulfite sequencing $)^{50}$ may reveal further CpG-SNP associations. (4) Liver tissue consists of multiple cell types that may have different methylation profiles, and we recognize that we cannot determine from which cell type the identified allelic imbalances originates using this study design. (5) Additionally, it should be emphasized that the results were generated in samples from subject with European ancestry, and our findings many not be generalizable to populations of different ethnicity.

In conclusion, we identified 112 genetic variants associated with DNA methylation in hemostatic genes in human liver tissue. Of these ASM-SNPs, 78\% were associated with expression of their respective genes, or with their respective protein levels or activity, or with other relevant hemostatic GWAS traits, suggesting that methylation regulated by genetic variants may provide a mechanistic link between noncoding SNPs and variation observed in circulating hemostatic proteins, prothrombotic diseases, and drug response. Our study highlights the importance of interaction analysis between genetic and epigenetic variation in relevant tissues, and that this approach can contribute to new insights into the biological processes affecting thrombosis and hemostasis.

\section{What is known about this topic?}

- Characterizing the complex relationship between genetic, epigenetic (e.g., DNA methylation), and transcriptomic variation has the potential to increase understanding about the mechanisms regulating hemostasis and inform drug development.

- Several hemostatic factors are synthesized in the liver. Despite DNA methylation being the most studied epigenetic trait, most studies are based on arrays that cover $<4 \%$ of $\mathrm{CpG}$ sites. Thus, no high-resolution information on DNA methylation is currently available for hemostatic genes in liver.

- Single-nucleotide polymorphisms (SNPs) can exert an influence on DNA methylation in cis which can affect gene expression. This can be analyzed through allelespecific methylation (ASM) experiments.

\section{What does this paper add?}

- We used a targeted bisulfite-sequencing approach to allow for the unbiased detection of all CpGs across 35 hemostatic genes and present the first high-resolution DNA methylation map for these genes in human liver.

- We identified 112 candidate ASM-SNPs in 24 hemostatic genes, all representing novel genotype-methylation associations in liver tissue. Of these SNPs, 76 were previously associated with gene expression and 61 were previously associated with circulating hemostatic proteins, relevant complex traits (e.g., coronary artery disease, stroke, venous thromboembolism), and drug dosing (e.g., warfarin).

- This suggests that methylation regulated by SNPs in cis may provide a mechanistic link between noncoding SNPs and variation observed in hemostatic gene expression, circulating hemostatic proteins, prothrombotic diseases, and drug response.

\section{Authors' Contributions}

M.O.L., T.M.S., and C.J. conceived the research design of the present study. C.J. provided funding and was responsible for sample contribution. M.O.L. and S.K. isolated gDNA and RNA and M.O.L. prepared sequencing libraries. M.O.L., L.H., and S.N. performed statistical analyses. M.O.L., T.M.S., and S.K. drafted the figures. M.O.L., C.J., and T.M.S. interpreted the data. M.O.L. and T.M.S. drafted the manuscript. L.H., M.D.L., S.K., and C.J. intellectually reviewed the manuscript. All authors contributed to the last revision process and approved the version to be published.

\section{Funding}

This study was supported by the Swedish Heart and Lung Foundation (20160316), the Swedish Research Council (2018-02543), the Swedish state under the agreement between the Swedish government and the county 
councils (the ALF-agreement, ALFGBG-720081), the Swedish Foundation for Strategic Research (RIF14-0081), the Rune and Ulla Amlövs Foundation for Neurologic Research, the John and Brit Wennerström Foundation for Neurologic Research, the Marcus Borgströms Foundation for Neurologic Research, and the Nilsson-Ehle Endowments.

\section{Conflict of Interest}

None declared.

\section{Acknowledgments}

The authors thank the Transplant Centre, Sahlgrenska University Hospital for technical and administrative assistance in providing the liver specimens. We thank the sequencing service provided by Genomics Core Facility at the University of Gothenburg, and Science for Life Laboratory (SciLifeLab), Stockholm. The computations were performed using resources provided by the Swedish National Infrastructure for Computing (SNIC) through Uppsala Multidisciplinary Center for Advanced Computational Science (UPPMAX). The authors gratefully acknowledge support from the Bioinformatics Long-term Support, SciLifeLab (WABI, Stockholm and Uppsala, Sweden) for RNA sequencing data analysis.

\section{References}

1 Kerkel K, Spadola A, Yuan E, et al. Genomic surveys by methylation-sensitive SNP analysis identify sequence-dependent allelespecific DNA methylation. Nat Genet 2008;40(07):904-908

2 Zhang Y, Rohde C, Reinhardt R, Voelcker-Rehage C, Jeltsch A. Nonimprinted allele-specific DNA methylation on human autosomes. Genome Biol 2009;10(12):R138

3 Schalkwyk LC, Meaburn EL, Smith R, et al. Allelic skewing of DNA methylation is widespread across the genome. Am J Hum Genet 2010;86(02):196-212

4 Paliwal A, Temkin AM, Kerkel K, et al. Comparative anatomy of chromosomal domains with imprinted and non-imprinted allelespecific DNA methylation. PLoS Genet 2013;9(08):e1003622

5 Goll MG, Bestor TH. Eukaryotic cytosine methyltransferases. Annu Rev Biochem 2005;74:481-514

6 Robertson KD. DNA methylation and human disease. Nat Rev Genet 2005;6(08):597-610

7 Lister R, Pelizzola M, Dowen RH, et al. Human DNA methylomes at base resolution show widespread epigenomic differences. Nature 2009;462(7271):315-322

8 Brenet F, Moh M, Funk P, et al. DNA methylation of the first exon is tightly linked to transcriptional silencing. PLoS One $2011 ; 6(01)$ : e14524

9 Aran D, Toperoff G, Rosenberg M, Hellman A. Replication timingrelated and gene body-specific methylation of active human genes. Hum Mol Genet 2011;20(04):670-680

10 Jjingo D, Conley AB, Yi SV, Lunyak VV, Jordan IK. On the presence and role of human gene-body DNA methylation. Oncotarget 2012; 3(04):462-474

11 Wagner JR, Busche S, Ge B, Kwan T, Pastinen T, Blanchette M. The relationship between DNA methylation, genetic and expression inter-individual variation in untransformed human fibroblasts. Genome Biol 2014;15(02):R37

12 Yang X, Han H, De Carvalho DD, Lay FD, Jones PA, Liang G. Gene body methylation can alter gene expression and is a therapeutic target in cancer. Cancer Cell 2014;26(04):577-590

13 Kurdyukov S, Bullock M. DNA methylation analysis: choosing the right method. Biology (Basel) 2016;5(01):3
14 Kint S, De Spiegelaere W, De Kesel J, Vandekerckhove L, Van Criekinge W. Evaluation of bisulfite kits for DNA methylation profiling in terms of DNA fragmentation and DNA recovery using digital PCR. PLoS One 2018;13(06):e0199091

15 Grundberg E, Meduri E, Sandling JK, et al; Multiple Tissue Human Expression Resource Consortium. Global analysis of DNA methylation variation in adipose tissue from twins reveals links to disease-associated variants in distal regulatory elements. Am J Hum Genet 2013;93(05):876-890

16 Kaplow IM, MacIsaac JL, Mah SM, McEwen LM, Kobor MS, Fraser HB. A pooling-based approach to mapping genetic variants associated with DNA methylation. Genome Res 2015;25(06):907-917

17 Kang EY, Martin LJ, Mangul S, et al. Discovering single nucleotide polymorphisms regulating human gene expression using allele specific expression from RNA-seq data. Genetics 2016;204(03): 1057-1064

18 Olsson Lindvall M, Hansson L, Klasson S, Davila Lopez M, Jern C, Stanne TM. Hemostatic genes exhibit a high degree of allelespecific regulation in liver. Thromb Haemost 2019;119(07): 1072-1083

19 Langmead B, Salzberg SL. Fast gapped-read alignment with Bowtie 2. Nat Methods 2012;9(04):357-359

20 Staley JR, Blackshaw J, Kamat MA, et al. PhenoScanner: a database of human genotype-phenotype associations. Bioinformatics 2016;32(20):3207-3209

21 GTEx Consortium. Human genomics. The Genotype-Tissue Expression (GTEx) pilot analysis: multitissue gene regulation in humans. Science 2015;348(6235):648-660

22 Battle A, Brown CD, Engelhardt BE, Montgomery SB; GTEx Consortium; Laboratory, Data Analysis \&Coordinating Center (LDACC) -Analysis Working Group; Statistical Methods groups-Analysis Working Group; Enhancing GTEx (eGTEx) groups; NIH Common Fund; NIH/NCI; NIH/NHGRI; NIH/NIMH; NIH/NIDA; Biospecimen Collection Source Site-NDRI; Biospecimen Collection Source Site-RPCI; Biospecimen Core Resource-VARI; Brain Bank Repository-University of Miami Brain Endowment Bank; Leidos Biomedical-Project Management; ELSI Study; Genome Browser Data Integration \&Visualization-EBI; Genome Browser Data Integration \&Visualization-UCSC Genomics Institute, University of California Santa Cruz; Lead analysts; Laboratory, Data Analysis \&Coordinating Center (LDACC); NIH program management; Biospecimen collection; Pathology; eQTL manuscript working group. Genetic effects on gene expression across human tissues. Nature 2017;550(7675):204-213

23 Eicher JD, Landowski C, Stackhouse B, et al. GRASP v2.0: an update on the Genome-Wide Repository of Associations between SNPs and phenotypes. Nucleic Acids Res 2015;43(Database issue): D799-D804

24 Franzén O, Ermel R, Cohain A, et al. Cardiometabolic risk loci share downstream cis- and trans-gene regulation across tissues and diseases. Science 2016;353(6301):827-830

25 Stanne TM, Olsson M, Lorentzen E, et al. A genome-wide study of common and rare genetic variants associated with circulating thrombin activatable fibrinolysis inhibitor. Thromb Haemost 2018;118(02):298-308

26 Olsson M, Stanne TM, Pedersen A, et al. Genome-wide analysis of genetic determinants of circulating factor VII-activating protease (FSAP) activity. J Thromb Haemost 2018;16(10):2024-2034

27 Gaunt TR, Shihab HA, Hemani G, et al. Systematic identification of genetic influences on methylation across the human life course. Genome Biol 2016;17:61

28 Ward LD, Kellis M. HaploReg v4: systematic mining of putative causal variants, cell types, regulators and target genes for human complex traits and disease. Nucleic Acids Res 2016;44(D1): D877-D881

29 Kundaje A, Meuleman W, Ernst J, et al; Roadmap Epigenomics Consortium. Integrative analysis of 111 reference human epigenomes. Nature 2015;518(7539):317-330 
30 Teichert M, Eijgelsheim M, Uitterlinden AG, et al. Dependency of phenprocoumon dosage on polymorphisms in the VKORC1, CYP2C9, and CYP4F2 genes. Pharmacogenet Genomics 2011;21 (01):26-34

31 Peden JFHJ, Saleheen D, Chambers JC, et al; Coronary Artery Disease (C4D) Genetics Consortium. A genome-wide association study in Europeans and South Asians identifies five new loci for coronary artery disease. Nat Genet 2011;43(04):339-344

32 Ikram MA, Seshadri S, Bis JC, et al. Genome-wide association studies of stroke. N Engl J Med 2009;360(17):1718-1728

33 Do C, Shearer A, Suzuki M, et al. Genetic-epigenetic interactions in cis: a major focus in the post-GWAS era. Genome Biol 2017;18(01):120

34 Kulis M, Heath S, Bibikova M, et al. Epigenomic analysis detects widespread gene-body DNA hypomethylation in chronic lymphocytic leukemia. Nat Genet 2012;44(11):1236-1242

35 Varley KE, Gertz J, Bowling KM, et al. Dynamic DNA methylation across diverse human cell lines and tissues. Genome Res 2013;23 (03):555-567

36 Hannon E, Spiers H, Viana J, et al. Methylation QTLs in the developing brain and their enrichment in schizophrenia risk loci. Nat Neurosci 2016;19(01):48-54

37 Gutierrez-Arcelus M, Lappalainen T, Montgomery SB, et al. Passive and active DNA methylation and the interplay with genetic variation in gene regulation. eLife 2013;2:e00523

38 Gutierrez-Arcelus M, Ongen H, Lappalainen T, et al. Tissue-specific effects of genetic and epigenetic variation on gene regulation and splicing. PLoS Genet 2015;11(01):e1004958

$39 \mathrm{Fu}$ J, Wolfs MG, Deelen P, et al. Unraveling the regulatory mechanisms underlying tissue-dependent genetic variation of gene expression. PLoS Genet 2012;8(01):e1002431

40 Bettscheider M, Kuczynska A, Almeida O, Spengler D. Optimized analysis of DNA methylation and gene expression from small, anatomically-defined areas of the brain. J Vis Exp 2012;(65):e3938
41 Gamazon ER, Badner JA, Cheng L, et al. Enrichment of cisregulatory gene expression SNPs and methylation quantitative trait loci among bipolar disorder susceptibility variants. Mol Psychiatry 2013;18(03):340-346

42 Do C, Lang CF, Lin J, et al. Mechanisms and disease associations of haplotype-dependent allele-specific DNA methylation. Am J Hum Genet 2016;98(05):934-955

43 Volkov P, Olsson AH, Gillberg L, et al. A genome-wide mQTL analysis in human adipose tissue identifies genetic variants associated with DNA methylation, gene expression and metabolic traits. PLoS One 2016;11(06):e0157776

44 Pirmohamed M, Burnside G, Eriksson N, et al; EU-PACT Group. A randomized trial of genotype-guided dosing of warfarin. $N$ Engl J Med 2013;369(24):2294-2303

45 Kimmel SE, French B, Kasner SE, et al; COAG Investigators. A pharmacogenetic versus a clinical algorithm for warfarin dosing. N Engl J Med 2013;369(24):2283-2293

46 Cullell N, Carrera C, Muiño E, Torres N, Krupinski J, FernandezCadenas I. Pharmacogenetic studies with oral anticoagulants. Genome-wide association studies in vitamin $\mathrm{K}$ antagonist and direct oral anticoagulants. Oncotarget 2018;9(49):29238-29258

47 Luo Z, Liu R, Sun B, et al. Identification of gene modules associated with warfarin dosage by a genome-wide DNA methylation study. Pharmazie 2018;73(05):288-293

48 Gallego-Fabrega C, Carrera C, Reny JL, et al. PPM1A methylation is associated with vascular recurrence in aspirin-treated patients. Stroke 2016;47(07):1926-1929

49 Cotto KC, Wagner AH, Feng YY, et al. DGIdb 3.0: a redesign and expansion of the drug-gene interaction database. Nucleic Acids Res 2018;46(D1):D1068-D1073

50 Yang Y, Scott SA. DNA methylation profiling using long-read single molecule real-time bisulfite sequencing (SMRT-BS). Methods Mol Biol 2017;1654:125-134 